Apidologie, 1977, 8 (1) 89-100.

\title{
NACHWEIS ÜBEREINSTIMMENDER CHROMOSOMEN-ZAHLEN (N = 16) BEI ALLEN 4 APIS-ARTEN *
}

\author{
Preuve du nombre identique de chromosomes chez les 4 espèces d'Apis \\ $(n=16)$ \\ Hartmut FAHRENHORST ** \\ Zoologisches Institut der Universität Münster/Westfalen
}

\section{SUMMARY}

Uniform Chromosome numbers ( $=16$ ) in the four species of Apis

Due to the contradictionary reports in the existing literature, a comparative study of the chromosomes in all the four species of Apis during the various stages of spermatogenesis was carried out. The testes of white-eyed drone pupae were employed in these studies. Maceration and evaporation techniques were used. Following hypotonic treatment the haploid meiotic chromosome number could be visualized nicely in all the four species.

The haploid chromosome number is 16 and this was found uniformly in Apis mellifica, $A$. cerana, $A$. dorsata and $A$. florea. As such the reported number of 8 haploid chromosomes in the last 2 species mentioned above should be rejected. It is doubtless, that in all the species of Apis, the diploid set of female germline cells consists of 32 chromosomes, as this was established for Apis mellifica.

As such, on the basis of the karyotype, no subdivision of the genus Apis is possible.

\section{ZUSAMMENFASSUNG}

Aufgrund der widersprüchlichen Literaturangaben und der unbefriedigenden Qualität des bislang veröffentlichten Bildmaterials wurde an allen 4 Apis-Arten eine vergleichende Untersuchung der Chromosomen in verschiedenen Spermatogenese-Stadien durchgeführt.

\footnotetext{
* Mit dankenswerter Unterstützung der Deutschen Forschungsgemeinschaft (Projekt Prof. Dr. W. Engels).

** Die Untersuchungen wurden im Rahmen eines Graduierten-Förderungs-Stipendiums durchgeführt.

Neue Anschrift : Institut für Biologie III (Zoologie) der Universität, Lehrstuhl Entwicklungsphysiologie, Auf der Morgenstelle 28, D-7400 Tübingen.
} 
Als Material wurden Testes von weißäugigen Drohnen-Puppen verwendet, die als Mazerations-Abdampf-Präparate aufgearbeitet wurden. Nach Hypoton-Vorbehandlung konnten die haploiden Meiose-Chromosomensätze der 4 Spezies gut dargestellt werden. Der haploide Satz besteht bei Apis mellifica, A. cerana, A. dorsata und A. florea übereinstimmend aus 16 Chromosomen. Die Mitteilungen über das Vorkommen von haploid nur 8 Chromosomen bei den beiden letztgenannten Arten müssen damit als widerlegt angesehen werden. Es besteht kein Zweifel an der Annahme, daß bei allen Apis-Arten der diploide Satz in weiblichen KeimbahnZellen aus 32 Chromosomen bestehen dürfte, wie dies für Apis mellifica nachgewiesen ist. Vom Karyotyp her kann somit keine Untergliederung des Genus Apis vorgenommen werden.

\section{EINLEIT UNG}

Die ersten karyologischen Untersuchungen an Honigbienen wurden schon zu Beginn des Jahrhunderts von Meves (1907), Doncaster (19061907), Armbruster (1913) und Nachtsheim (1913) an Apis mellifica durchgeführt. Die Autoren beschrieben in der männlichen Keimbahn einen haploiden Satz von 16 Chromosomen und in der weiblichen einen diploiden von 32. Die Kleinheit der Bienenchromosomen erschwerte die Analysen, so daß weitere Einzelheiten erst von Sanderson und Hall (1948) mitgeteilt wurden. Sie konnten unter den 16 Chromosomen ein großes, hakenförmiges identifizieren, das von Manning (1949) als X-Chromosom bezeichnet wurde. Da3 es sich hierbei jedoch nicht um ein Geschlechtschromosom handeln kann, wurde bereits 1952 von Ris und Kerr gezeigt. Die von Meves, Armbruster und Naснтsнеiм bei Apis mellifica gefundenen Chromosomenzahlen wurden in einer umfassenden Nachuntersuchung von WoLF (1960) bestätigt.

Cytologische Studien an den 3 weiteren Honigbienen-Arten, deren Vorkommen auf das tropische Asien beschränkt ist, wurden erst wenige Male in letzter Zeit und vorzugsweise von indischen Autoren durchgeführt. Deodikar, Thakar und Shah beschrieben 1959 für Apis cerana einen haploiden Chromosomensatz von 16, was 1961 von Sharma, Gupta und Kumbkarni bestätigt wurde. Widersprüchlich sind die Befunde über Chromosomenzahlen der beiden anderen Arten. Bei Apis dorsata wurden von KumbKarni (1963) 16, von Deodikar und Thakar (1966) dagegen 8 Chromosomen im haploiden Satz ermittelt. Die gleiche Zahl $n=8$ gaben Thakar und Deodikar (1966) für Apis florea an.

Kerr (1969) stellte aufgrund dieser Ergebnisse eine Theorie über phylogenetische Polyploidisierung im Verlauf der Evolution der Apiden auf, die er später auch auf verschiedene Meliponen-Arten ausdehnte (KERr, 1974).

Zur Klärung dieser widersprüchlichen Literatur-Angaben über die Chromosomen-Zahlen im Genus Apis überprüfte ich mit Hilfe neuerer Methoden und Hilfsmittel (Mazerations-Technik, spezifische Färbungen, Phasenkontrast-Mikroskopie) den Karyotyp aller 4 rezenten Apis-Arten. Als Untersuchungsmaterial wurden Stadien der männlichen Meiose gewählt. 


\section{MATERIAL UND METHODEN}

\section{Tiermaterial}

Späte Larvenstadien und weißäugige Drohnenpuppen. Die Apis mellifica-Drohnen wurden Standvölkern des Instituts (mellifica-carnica-Hybriden) entnommen. Das Material der 3 tropischen Honigbienen-Arten $A$. cerana, $A$. dorsata und $A$. florea wurde in Ceylon während eines Arbeitsaufenthaltes an der Universität Peradeniya gesammelt und fixiert. Ein Teil der florea-Puppen wurden mir dankenswerterweise von Herrn Dr. Koeniger (Oberursel) aus Ceylon mitgebracht.

\section{Präparation}

Die Drohnenwabenstücke mit den gewünschten Larven-und Puppen-Stadien wurden stets erst unmittelbar vor der Präparation den Völkern entnommen. Mit feinen Pinzetten wurden die Abdomina unter Insekten-Ringer dorsal eröffnet. Bei Larven und Puppen sind die Hodenschläuche die größten Organe, sie sind wie der Darm von vielen Fettkörperzellen umgeben. Um sie zu entfernen, wurden die Hoden freipräpariert und kurz in Ringer gewaschen.

\section{Hypoton-Behandlung}

Um die bei Honigbienen sehr kleinen Chromosomen besser darstellen zu können, wurden sie durch Hypoton-Behandlung aufgequollen. Dazu wurde das frisch präparierte Hodengewebe für $1 / 2$ bis $1 \mathrm{~h}$ in OHNUKI-Lösung (1965) überführt. Dieses optimale Verfahren konnte, weil Labor-gebunden, nur bei $A$. mellifica und $A$. cerana angewandt werden. Bei $A$. dorsata und $A$. florea wurde ein gewisser Quellungsgrad der bereits fixierten Chromosomen dadurch erreicht, daß sie über längere Zeit in Orcein-Milchsäure-Lösung angefärbt wurden.

\section{Fixierung}

Für ca. $12 \mathrm{~h}$ in Carnoy's Gemisch (Aethanol/Eisessig $3: 1$ bei $8-10$ "C). Bis zur weiteren Verarbeitung wurden die Testes sodann in reinem Isopropanol bei -20 "C gelagert.

\section{Mazeration}

In einer kleinen Menge des Fixierungsgemisches wurden die Testes sehr fein zerzupft, in $50 \%$ iger Essigsäure aufgenommen und darin ca. 2 min. mazeriert. Anschließend wurden Proben mit der Pipette bei ca. $40{ }^{\circ} \mathrm{C}$ im Trockenschrank auf schrägliegende Objektträger aufgetropft, bis diese zu $1 / 3$ bis $1 / 2$ bedeckt waren. Bei der folgenden Trocknung bildeten sich Niederschlagsringe, in denen neben Zellkernen und Cytoplasmaresten freie Meioseund Mitose-Platten zu finden waren (vgl. WEBER, 1966).

\section{Färbung}

Mit Orcein-Milchsäure unmittelbar vor der mikroskopischen Untersuchung.

\section{Mikrophotographie}

Mit dem Zerss-Photomikroskop II und Ölimmersionen $100 \times$ (Neofluare, Planapochromate, Apochromate) im Hellfeld und Phasenkontrast.

\section{ERGEBNISSE}

Die männliche Meiose von Apis ist eine Einschritt-Pseudo-Meiose ohne Reduktion des in der männlichen Keimbahn ohnehin haploiden Chromosomensatzes (Doncaster, 1906; Wolf, 1960). Die erste meiotische Teilung 
fällt aus. Bis zur Metaphase der einzigen, eigentlich zweiten meiotischen Teilung sind somit in allen Stadien stets aus zwei Schwesterchromatiden bestehende Univalente vorhanden.

Die artspezifische Anzahl der im haploiden Satz vorliegenden Chromosomen kann im Spermatogenese-Verlauf am sichersten durch Auszählen dieser Univalente bestimmt werden. Als hierfür günstigste Stadien erwiesen sich die Diakinese (Abb. 1) und die Metaphase II (Abb. 2 u. 3). Die Chromosomen-Individualität ist nach Hypoton-Behandlung frischpräparierten Hodenmaterials (Abb. 1 u. 2) wesentlich hesser zu erkennen als nach Anfärben vorfixierten Materials (Abb. 3 u. 4). Mit beiden Techniken kann jedoch die Zahl der vorliegenden Univalente zweifelsfrei ermittelt werden. Für alle 4 rezenten Honigbienen-Arten, d. h. Apis mellifica, Apis cerana, Apis dorsata und Apis florea wurden übereinstimmende Chromosomenzahlen festgestellt; der haploide Satz besteht immer aus $n=16$ Chromosomen (Abb. 1 - 4), wie dies für Apis mellifica (Abb. 1) bereits seit Meves (1907) und Doncaster $(1906,1907)$ bekannt ist. Die hier mitgeteilten Befunde über die 3 tropischen Apis-Arten stützen sich auf eine große Zahl entsprechender Beobachtungen, die absolut reproduzierbar an Hodenmaterial von vielen Drohnenpuppen und teils verschiedener Herkünfte gemacht werden konnten.

Die nunmehr nachgewiesene zahlenmäßige Übereinstimmung der Chromosomensätze aller vier Apis-Arten läßt erwarten, daß interspezifische Genom-Homologien zu finden sein sollten. Ein Vergleich zeigt, daß ein auffallend großes Chromosom in sämtlichen Sätzen vorkommt. Es ist in den Abbildungen 1 - 4 mit einem großen Pfeil markiert. In einzelnen Präparaten (vgl. Abb. 2 u. 3) ist auch das Muster der heterochromatischen Bereiche auf den beiden etwas unterschiedlich langen Schenkeln dieses großen Chromosoms zu erkennen, es stimmt bei allen vier Arten weitgehend überein. Weiterhin enthalten alle Sätze ein besonders kleines, insgesamt stark heterochromatisches Chromosom, das in den Abb. 1 - 4 mit einem kleinen Pfeil bezeichnet ist. Die restlichen 14 Chromosomen sind von mittlerer Größe und teils akrozentrisch, teils mesozentrisch. Über sie kann durch Bildvergleich allein keine Aussage über eventuelle weitere interspezifische Homologien gemacht werden.

Bereits in der Anaphase (Abb. 5) kommt es zu einer Kontraktion der nur noch aus einer Chromatide bestehenden Chromosomen, deren Individualität in der Telophase (Abb. 6) noch undeutlicher wird. In diesen Stadien sind Auszählungen der vorliegenden haploiden Chromosomenzahlen daher äußerst schwierig, sie führen häufig zu falschen, und zwar zu zu niedrigen Ergebnissen, weil außerdem die auch in somatischen Interphasekernen ausgeprägte Tendenz zur Chromozentren-Bildung wieder einsetzt.

Seit den Untersuchungen von RIsLer (1954) über Spindelgrößen ist bekannt, daß im Soma von Drohnen der haploide Zustand in der Embryoge- 
nese bald aufgegeben wird und je nach Gewebe diploide bzw. auch polyploide Zellen entstehen (vgl. Risler und Romer, 1968). Diese Aussagen stützen sich durchweg auf cytophotometrische Bestimmungen des DNS-Gehaltes (Merriam et al., 1954; Mello u. Takahashi, 1971; Melnitschenko u. Kapralowa, 1971). Es war bisher nicht bekannt, ob in solchen oligoploid gewordenen Kernen etwa in Endometaphasen Einzelchromosomen dargestellt und ggf. auch Chromosomenzahlen bestimmt werden können. Dies ist, wie am Beispiel Apis cerana die Abb. 7 u. 8 zeigen, tatsächlich der Fall : Endometaphaseplatten aus nicht näher bestimmten somatischen Hodenzellen lassen in Mazerationspräparaten den diploiden (Abb. $7: \mathbf{n}=32$ ) bzw. oktoploiden (Abb. $8: \mathbf{n}=128$ ) Status erkennen.

\section{DISK USSION}

Arrhenotokie ist der bei fast allen Hymenopteren realisierte Mechanismus der Geschlechtsbestimmung (Whiting, 1943, Ruttner, 1966). Es handelt sich hierbei um ein haplo-diploides System, das nach den Vorstellungen von Kerr und Silveira (1972) wahrscheinlich aus einem diplodiploiden System und nicht durch Polyploidisierung aus einem haplohaploiden System entstanden sein dürfte. Es stellt sich damit die Frage, ob die Karyotypen heute lebender Arten entsprechende Hinweise bieten.

Die ursprünglich von Kerr (1952) aufgestellte Hypothese einer Polyploidisierung in der Phylogenie der sozialen Hymenopteren hat nach der Analyse entsprechender chromosomaler Zahlenverhältnisse bei Halictiden, Trigoniden und Meliponiden zu verschiedenen Stammbaum-Konstruktionen für Bienen geführt (Kerr, 1969; Kerr und Silveira, 1972), die nicht in allen Punkten z. B. mit Michener's (1974) Schema einer sozialen Evolution bei den Apoidea übereinstimmen. In die Hypothesen über Karyotyp-Evolution paßten bisher auch die karyologischen Daten der Gattung Apis. Sie wurde nach den ermittelten Chromosomen-Zahlen in 2 Gruppen von je 2 Arten aufgeteilt $: \mathbf{n}=8$ Chromosomen bei Apis florea (Thakar und Deodikar, 1966) und Apis dorsata (Deodikar und Thakar, 1966) sowie $\mathrm{n}=16$ Chromosomen bei Apis cerana (Deodrkar, Тhakar und Shaн, 1959) und Apis mellifica (Meves, 1907; Armbruster, 1913; Nachtsheim, 1913; Wolf, 1960). Für Apis mellifica (Nachtsheim, 1913) und Apis cerana (Deodikar und Thakar, 1966) wurde außerdem das Auftreten von Tetraden in der männlichen und Oktaden in der weiblichen Meiose beschrieben. Dies führte zu der Annahme einer Paarung ursprünglich homologer Elemente innerhalb des jetzt haploiden Satzes. Im gleichen Sinn interpretierte Wouf (1960) das Auftreten von 7-8 Chromozentren in Somazellen junger Drohnenpuppen 


\section{TAFEL I}

Aвв. 1-4. - Die zahlenmäßig übereinstimmenden männlichen Chromosomen-Sätze der 4 rezenten Honigbienen-Arten mit jeweils 16 Chromosomen, die vor der einzigen meiotischen Teilung je aus 2 zusammenhängenden Chromatiden bestehen.

Mit Pfeilen sind offensichtlich homologe Einzelchromosomen gekennzeichnet : ein sehr großes und $\longrightarrow$ ein kleines, stark heterochromatisches Chromosom.

Aвв. 1. - Haploider Chromosomensatz von Apis mellifica mit $\mathrm{n}=16$ Chromosomen. Spermatogenese : Diakinese. Orcein-Milchsäure-Färbung nach Hypoton-Vorbehandlung. Phasenkontrast, $3600 \mathrm{x}$.

Aвв. 2. - Haploider Chromosomensatz von Apis cerana mit $\mathrm{n}=16$ Chromosomen. Spermatogenese : Metaphase II. Orcein-Milchsäure-Färbung nach Hypoton-Vorbehandlung. Durchlicht, $3000 \mathrm{x}$.

Aвв. 3. - Haploider Chromosomensatz von Apis dorsata mit $\mathrm{n}=16$ Chromosomen. Spermatogenese : Metaphase II. Orcein-Milchsäure-Färbung. Durchlicht, $3600 \mathrm{x}$.

Aвв. 4. - Haploider Chromosomensatz von Apis florea mit $\mathbf{n}=16$ Chromosomen. Spermatogenese : Metaphase II. Orcein-Milchsäure-Färbung. Durchlicht, $2800 \mathrm{x}$.

\section{PLANChe I}

FIG. 1-4. - Garniture chromosomiques mâles numériquement uniformes des 4 espèces actuelles d'abeille mellifique, chacun d'eux étant de 16 chromosomes qui se composent, avant la division méiotique unique, de 2 chromatides liées.

Les flèches indiquent des chromosomes haploïdes manifestement homologues : $\longrightarrow \longrightarrow$ un très gros chromosome et $\longrightarrow$ un petit, fortement hétérochromatique.

FIg. 1. - Garniture chromosomique haploïde d'Apis mellifica à $n=16$ chromosomes. Spermatogenèse : diacinèse. Coloration à l'acide lactique-orcéine après traitement hypotonique. Contraste de phase, $\times 3600$.

FIg. 2. - Garniture chromosomique haploïde d'Apis cerana à $n=16$ chromosomes. Spermatogenèse : métaphase II. Coloration à l'acide lactique-orcéine après traitement hypotonique. Lumière transmise, x 3000 .

Fıg. 3. - Garniture chromosomique haploïde d'Apis dorsata à $\mathrm{n}=16$ chromosomes. Spermatogenèse : métaphase II. Coloration à l'acide lactique-orcéine. Lumière transmise, × 3600 .

Fig. 4. - Garniture chromosomique haploïde d'Apis florea à n $=16$ chromosomes. Spermatogenèse : métaphase II. Coloration à l'acide lactique-orcéine. Lumière transmise, x 2800 . 

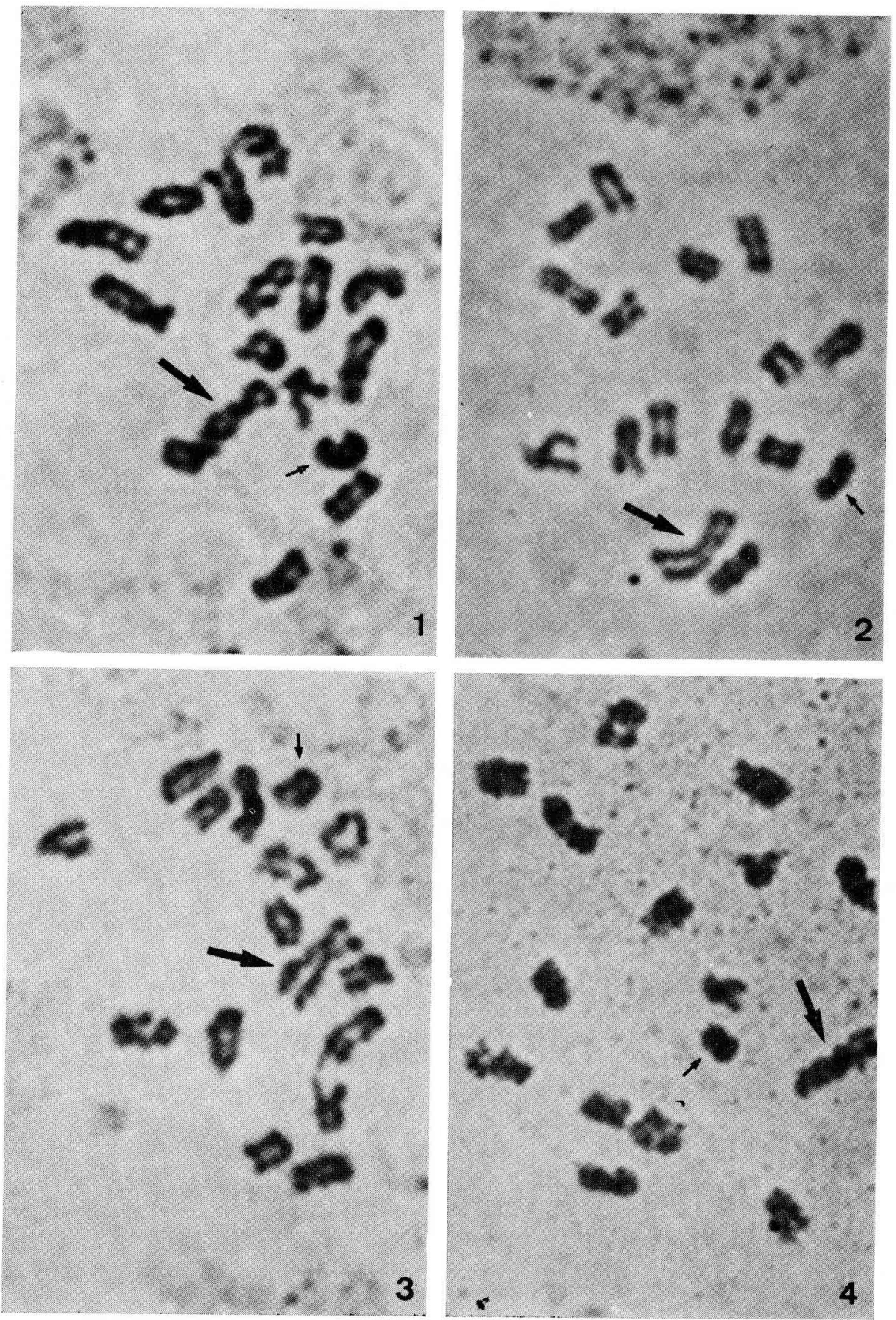

Hartmut FAHRENHORST 


\section{TAFEL II}

Aвв. 5. - Späte Anaphase II der Spermatogenese von Apis florea. Orcein-Milchsäure-Färbung. Durchlicht, $2800 \mathrm{x}$.

Авв. 6. - Frühe Telophase II der Spermatogenese von Apis cerana. Orcein-MilchsäureFärbung. Durchlicht, $1900 \mathrm{x}$.

Aвв. 7. - Somatisch polyploider männlicher Chromosomensatz von Apis cerana aus einer mesodermalen Zelle des Hodengewebes mit 32 Chromosomen, d. h. einem diploiden Genom. Orcein-Milchsäure-Färbung nach Hypoton-Vorbehandlung. Durchlicht, $3000 \mathrm{x}$.

Авв. 8. - Somatisch polyploider Chromosomensatz von Apis cerana aus einer mesodermalen Zelle des Hodengewebes mit 128 Chromosomen, d. h. einem oktoploiden Genom. OrceinMilchsäure-Färbung nach Hypoton-Vorbehandlung. Durchlicht, 3000 x.

\section{PLANCHE II}

Fig. 5. - Fin de l'anaphase II de la spermatogenèse d'Apis florea. Coloration à l'aice lactiqueorcéine. Lumière transmise, $\times 2800$.

FIG. 6. - Début de la télophase II de la spermatogenèse d'Apis cerana. Coloration à l'acide lactique-orcéine. Lumière transmise, x 1900.

FIG. 7. - Garniture chromosomique mâle polyplö̈dique somatique d'Apis cerana provenant d'une cellule mésodermique du tissu testiculaire et comportant 32 chromosomes, c'est-àdire un genôme diploïde. Coloration à l'acide lactique-orcéine après traitement hypotonique. Lumière transmise, $x 3000$.

FIg. 8. - Garniture chromosomique polyploïdique somatique d'Apis cerana provenant d'une cellule mésodermique du tissu testiculaire et comportant 128 chromosomes, c'est-à-dire une génôme octoploïdique. Coloration à l'acide lactique-orcéine. Lumière transmise, x 3000 . 

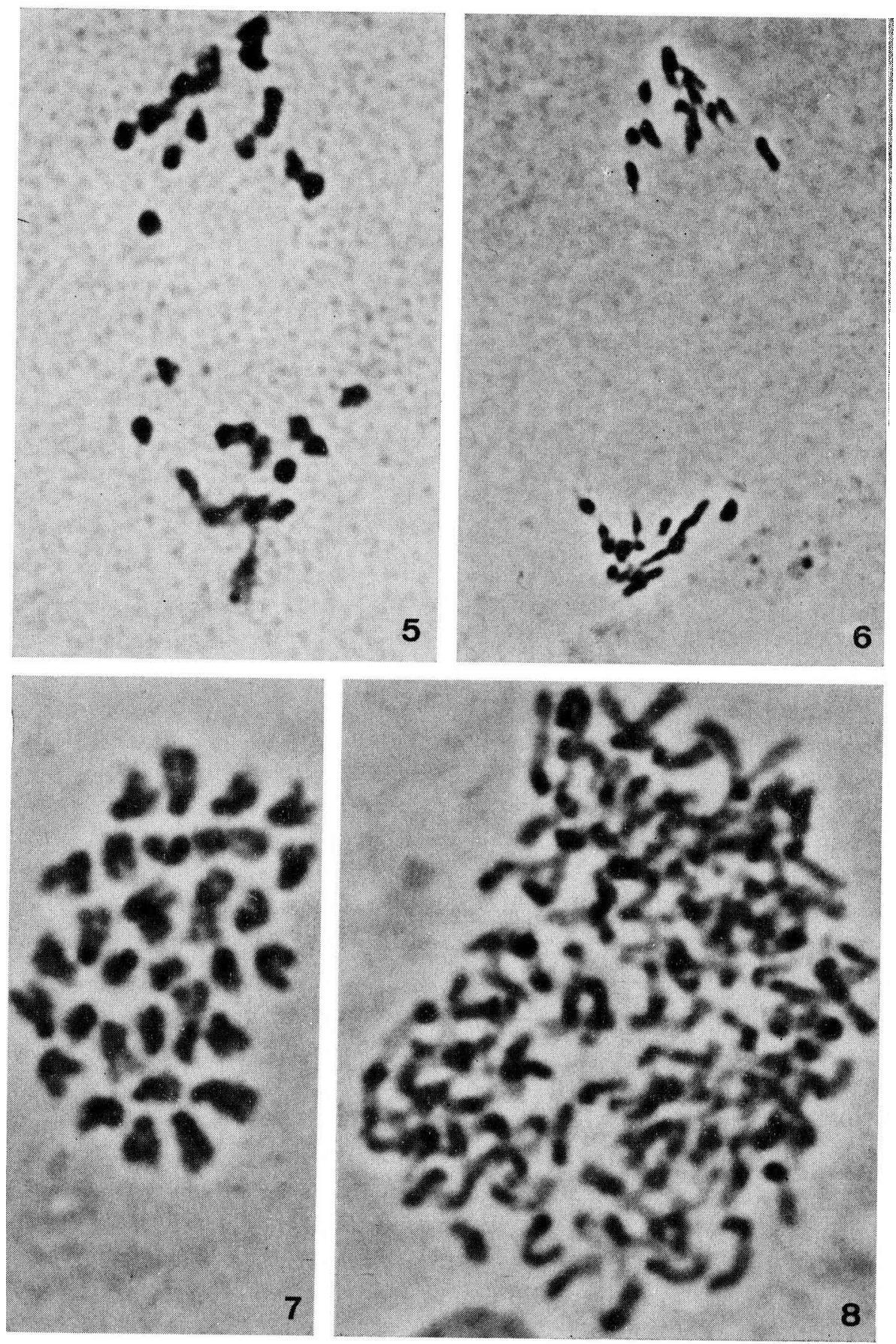
als “ Resthomologien », die auf 8 als ehemalige Grundzahl des Apis-Genoms schließen lassen könnte.

Hinweise für eine phylogenetische Polyploidisierung im Laufe der Stammesgeschichte liegen somit auch für das Genus Apis durchaus vor, wenn auch nach meinen Untersuchungen nunmehr feststeht, daß die Chromosomenzahl bei den heute lebenden Arten in keinem Fall mehr $\mathbf{n}=\mathbf{8}$ beträgt. Zu fragen ist, wie die publizierten Beobachtungen solcher Karyotyp-Werte in haploiden Keimbahnzellen gegebenenfalls zu erklären sind. Eine kritische Durchsicht der Originalliteratur läßt gewisse methodische Mängel bei manchen der von indischen Autoren durchgeführten Untersuchungen erkennen. Aus verschiedenen Gründen ist nicht auszuschließen, daß bei der Auszählung der äuBerst kleinen Apis-Chromosomen Irrtïmer unterlaufen sein können. Sofern es sich bei dem veröffentlichten Bildmaterial der fraglichen Chromosomen-Sätze überhaupt um. Mikrophotos und nicht um Zeichnungen handelt (vgl. Deodikar et al., 1959, Thakar und Deodikar, 1966; Deodikar und Thakar, 1966; KumbKarni, 1963), sind nicht immer Meiose-Stadien mit einwandfrei auszählbaren Chromosomen wiedergegeben worden. Dies dürfte bei Schnittpräparaten stets Schwierigkeiten bereiten (vgl. Fyg, 1973). Bei allen Apis-Arten verläuft die männliche Meiose als Pseudomeiose nach folgendem, insbesondere von WoLf (1960) eingehend belegtem Schema : Die Spermatocyten führen nur eine einzige, äquationelle Teilung durch (vgl. auch Doncaster, 1906; Fyg, 1973). Beide Spermatiden entwickeln sich, wenn auch mit einer mehrtägigen Phasenverzögerung (Kerr und Silveira, 1974), zu Spermien. In der einzigen Teilung werden dic zuvor deutlich aus 2 Chromatiden bestehenden Univalente aufgetrennt. Der Habitus der einzelnen Chromosomen ist nach der Anaphase der Einschritt-Pseudomeiose nicht mehr sicher zu identifizieren, in der Telophase kommt es bereits wieder zur Chromozentren-Bildung (vgl. Abb. 6) wie in somatischen Kernen. Zählfehler können bei der Analyse solch später Stadien daher leicht eine zu geringe, bei 8 liegende Chromosomenzahl vortäuschen.

Nachdem für alle 4 Apis-Arten nunmehr zweifelsfrei eine übereinstimmende Chromosomenzahl von $\mathrm{n}=16$ feststeht, kann aus dem numerischen Karyotyp keine unmittelhare Vorstellung einer trans-spezifischen GenomEvolution durch Polyploidisierung bei Honigbienen mehr abgeleitet werden. Die nahe Verwandtschaft sämtlicher rezenter Arten wird außerdem durch in allen 4 Chromosomensätzen erkennbare interspezifische Übereinstimmungen demonstriert. Sie sind vor allem für das große Chromosom (SANDerson und Hald, 1948; Wolf, 1960) evident. Ob noch weitere Homologien nachzuweisen sind, soll Gegenstand künftiger Untersuchungen sein. 


\section{RÉSUMÉ}

Les premières recherches caryologiques sur l'abeille datent du début du siècle. Elles montrèrent qu'Apis mellifica possédait 16 chromosomes pour la garniture mâle haploïde et 32 chromosomes pour la garniture femelle diploïde. La petitesse des chromosomes rendait difficile des recherches plus poussées. Ce n'est que beaucoup plus tard qu'on décrivit dans la formule chromosomique un grand élément en forme de crochet, que l'on désigna pendant quelque temps par chromosome sexuel. Mais la validité de cette dénomination fut par la suite démentie. Une recherche ultérieure détermina les conditions particulières de la méiose et les nombres de chromosomes chez Apis mellifica.

Les chromosomes des 3 espèces tropicales d'abeille mellifique ne furent étudiés que récemment, principalement par des chercheurs indiens, et l'on obtint des résultats contradictoires. On a trouvé pour Apis cerana n $=16$, ou $2 \mathrm{n}=32$ chromosomes, comme chez Apis mellifica; pour Apis dorsata, parfois $\mathrm{n}=16$, mais parfois aussi $\mathrm{n}=8$; et pour Apis florea, également $\mathbf{n}=8$. D'après quoi on divisa le genre Apis en deux groupes d'espèces et une théorie fut élaborée sur la polyploïdisation phylogénétique dans l'évolution des Apides.

En raison des données contradictoires de la littérature et l'absence de photographie univoque des formules chromosomiques des espèces tropicales d'abeille mellifique, nous avons mené une étude comparative des chromosomes chez le genre Apis. Nous avons utilisé comme matériel des testicules de pupes mâles au stade yeux blancs. Après un traitement hypotonique ou une fixation, on fait macérer le tissu testiculaire dans l'acide acétique à $50 \%$, évaporer, puis on monte les préparations sur porte-objectif. Les plaques chromosomiques obtenues sont teintées par le mélange acide lactique-orcéine. Les stades de diacinèse et de métaphase II de la méiose, au cours desquelles les chromosomes haploïdes se composent de deux chromatides, se prêtent particulièrement bien au Génombrement des chromosomes. On a pu prouver que chez les quatre espèces d'Apis le nombre de chromosomes haploïdes était uniforme et de 16 .

Une comparaison interspécifique permet en outre de reconnaître quelques homologies dans les chromosomes. Chez les quatre espèces il existe un chromosome particulièrement gros et un autre, petit et fortement hétérochromatique. Les 14 chromosomes restants sont d'une taille moyenne, en partie acrocentriques, en partie mésocentriques. Après l'unique division méiotique il se produit, au cours de l'anaphase II et particulièrement de la télophase, une contraction des chromosomes constitués à présent d'une chromatide. Il n'est done plus facile de les reconnaître individuellement, d'autant plus qu'ils ont tendance à former des chromocentres interphasiques.

Chez Apis cerana on a pu, à l'aide des mêmes techniques, décrire des plaques d'endométaphase de cellules somatiques polyploïdiques du tissu testiculaire.

Sur la base des résultats exposés ici, il faut considérer comme erronées les données de la littérature qui affectent un nombre $\mathrm{n}:=8$ chromosomes à Apis dorsata et Apis florea. Une subdivision du genre Apis selon le caryotype ne peut plus être envisagée en raison de l'uniformité chromosomique.

\section{LITERATURVERZEICHNIS}

Armbruster L., 1913, Ưher die Chromatinverhältnisse bei solitären Bienen und ihre Beziehung zur Frage der Geschlechtsbestimmung. Ber. Natur. Ges. Freiburg i. Br., 20, 4-12.

Deodikar G. B., Thakar C. V., 1966, Cyto-genetics of indian honeybee and bearing on taxonomic and breeding problems. Indian J. Genet., 26 A, 386-393. 
Deodikar G. B., Thakar C. V., Shah P. N., 1959, Cyto-genetic studies in Indian honey bees. Indian Acad. Sci., Proc. Section B 49, 194-206.

Doncaster L., 1906, Spermatogenesis of the hive bee (Apis mellifica). Anat. Anz., 29, 490-491.

Doncaster L., 1907, Spermatogenesis of the Honey Bee. Anat. Anz., 31, 168-169.

FyG W., 1973, Üher den Einfluß von Metamorphose-Häutungsstörungen und Ligaturen auf die Spermatogenese der Honigbiene (Apis mellifica L.). Apidologie, 4, 227-265.

KFнR W. E., 1952, A variação do nûmero de cromossomas na evolução dos Hymenoptera. Scientia Genética (Torino), 4, $182-190$.

KERR W. E., 1969, Some aspects of the evolution of social bees (Apidae). Evol. Biol., 3, $119-$ 175 .

KerR W. E., 1974, Advances in Cytology and Genetics of Bees. Annu. Rev. Entomol., 19 , 253-268.

KenR W. F., Silveira Z. V., 1972, Karyotypic evolution of the bees and corresponding taxonomic implications. Evolution, 26, 197-202.

Kerr W. E., Silverra Z. V., 1974, A note on the formation of honeybee spermatozoa. J. Apic. Res., 13, 121-126.

Kumbкarni C. G., 1963, Cytological studies in Hymenoptera : Part I - Cytology of parthenogenesis in the honey-bee, Apis dorsata. Indian J. Exp. Biol., 2, 65-68.

Manning F. J., 1949, Sex determination in the honey-bee. Microscope, 7, 175-180.

Mello M. L. S., TaKahashi C. S., 1971, DNA content and nuclear volume in larval organs of Apis mellifera. J. Apic. Res., 10, 125-132.

Melnitschenko A. N., Kapraiowa O. W., 1971, Der Zellenkaryotyp der somatischen Bienengewebe (Apis mellifica L.) in verschiedenen Ontogenese-Phasen. Proc. 23. Int. Apimondia-Congr. (Moskau), 385-390.

Merriam R. W., RIs H., 1954, Size and DNA content of nuclei in various tissues of male, female and worker honeybees. Chromosoma, 6, 522-538.

Meves F., 1907, Die Spermatocytenteilungen bei der Honjgbiene (Apis mellifica L.), nebst Bemerkungen über Chromatinreduktion. Arch. mikr. Anat. Entw. gesch., 70, 414-491.

Michener Ch. D., 1974, The social behaviour of bees The Belknap Press of Harvard University Press, Cambridge, Massachusetts.

Nachtsherm H., 1913, Cytologische Studien über die Geschlechtsbestimmung bei der Honigbiene (Apis mellifica L.). Arch. Zellforsch., 11,170-239.

OHNUKr Y., 1965, Demonstration of the spiral structure of human chromosomes. Nature (Lond.), 208, 916-917.

Risler H., 1954, Die somatische Polyploidie in der Entwicklung der Honigbiene (Apis mellifica L.) und die Wiederherstellung der Diploidie bei den Drohnen. Z. Zellforsch., 41, 1-78.

Risler H., Romer F., 1968, Chromosomenzahlen und DNS-Gehalt der Epidermiszellen während der Entwicklung der Honigbiene. Zool. Anz. Suppl., 32, 153-160.

Rutwner F., 1966, Welche Faktoren bestınımen das Geschlecht? Neue Ergebnisse der Studien an Hautflüglern. Umschau, 21, $093-696$.

Sanderson A. R., Hall D. W., 1948, The cytology of the honey-bee Apis mellifera L. Nature (Lond.), 162, 34.

Sharma G. P., Gupta B. L., Kumbearni C. G., 1961, Cytology of spermatogenesis in the Honey Bee, Apis indica (F.). J. Roy, Microscop. Soc., 79, 337-351.

Thakar C. V., Deodikar G. B., 1966, Chromosome number in Apis florea Fab., Current Science, 7, 186.

Weber F., 1966, Beitrag zur Karyotypanalyse der Laufkäfergattung Carabus L. (Coleoptera). Chromosoma (Berl.), 18, 467-476.

Whiting P. W., 1943, Multiple alleles in complementary sex determination of Habrobracon. Genetics, 28, 365-382.

Wolf B. E., 1960, Eine Nachuntersuchung zur Cytologie der Honigbiene (Apis mellifica L.). Zool. Beitr., 5, 373-391. 